\title{
Towards a practical inclusive design approach
}

\author{
Simeon Keates, P. John Clarkson and \\ Lee-Anne Harrison \\ University of Cambridge, Dept. of Engineering, \\ Trumpington Street, Cambridge. CB2 1PZ \\ United Kingdom \\ +441223766958 \\ lsk12, pjc10, ljh47@eng.cam.ac.uk
}

\section{ABSTRACT}

It is known that many products are not accessible to large sections of the population. Designers instinctively design for able-bodied users and are either unaware of the needs of users with different capabilities, or do not know how to accommodate their needs into the design cycle.

This aim of this paper is to present a methodological design approach for implementing inclusive design. A summary of the principal methods for designing for users with different capabilities is given along with a description of a model, the Inclusive Design Cube, that displays how the different approaches are complementary and can provide complete population coverage. A case study of the design of an information point for use in a post office, shall be used to highlight the use of the model.

\section{Keywords}

Case study, user-aware design, functional impairments

\section{INTRODUCTION}

Conventional product interfaces present serious difficulties to users with functional impairments. Conditions causing such impairments can occur throughout the life course, affecting all age groups. Certain symptoms, such as reduced hearing, appear with increasing frequency with advancing age, whilst other such as spasms are often associated with particular medical conditions, such as Cerebral Palsy. This has led to the common concept of "the disabled and elderly" as being groups requiring separate attention. Consequently, many design approaches for allowing accessibility by members of either group focus on their disabilities. However, the principal concern for a designer should be physical capabilities, irrespective of cause.

Currently, there is surprisingly little industry awareness of the benefits of inclusive design despite the inexorable growth of the global aging population. Hence, there is an urgent need for design methods, based on a better understanding of age and ability related factors, which will

Permission to make digital or hard copies of all or part of this work for personal or classroom use is granted without fee provided that copies are not made or distributed for profit or commercial advantage and that copies bear this notice and the full citation on the first page. To copy otherwisc, to republish, to post on servers or to redistribute to lists, requires prior specific permission and/or a fee. CUUJ' 00 Arlington VA USA

Copyright ACM 2000 1-58113-314-6/00/11 $\$ \$ 5.00$

\author{
Peter Robinson \\ University of Cambridge, Computer Laboratory, \\ Pembroke Street, Cambridge. CB2 3QG \\ United Kingdom \\ +441223334637 \\ Peter.Robinson@cl.cam.ac.uk
}

lead to a minimizing of the impact of impairments and thereby extend quality life.

\section{Designing for All}

During the past 20 years, considerable advances have been made in the fields of designing for the disabled or older user, with different approaches reflecting local cultural, political and historical situations. In the USA, the concept of 'Barrier-Free' design was developed in response to the demands of the disability movement. This led to Section 504 of the Rehabilitation Act (1973), prohibiting discrimination against people with disabilities; the Fair Housing Amendments Act (1988); and the Americans with Disabilities Act (1990) [2], which asserts the individual's right to use products and services on an equal access basis. In 1996, the UK implemented the Disability Discrimination Act [7] which covers similar ground to the ADA.

In these, and other recent initiatives, a growing unease with terminology such as the disabled and elderly can be seen with the expression Design for All now being used, for example, in European Commission documents.

\section{Universal Access and The Post Office}

The Post Office (TPO) is one of the UK's largest employers, directly employing some 190,000 people. It consists of four core businesses - Royal Mail; Parcelforce Worldwide; Post Office Counters; and Subscription Services Limited. Of particular interest in this paper is Post Office Counters, which is responsible for the operational management of and services provided by all major post offices.

As with all large UK companies, TPO is expected to meet the requirements of the 1996 Disability Discrimination Act [7]. This stipulates a minimum proportion of employees with physical impairments of $3 \%$ and that the work-place must not present unnecessary obstacles to employment opportunities. It also stipulates that all companies offering services to the public should not bar use of those services on the grounds of end-user disability.

The Post Office plays a pivotal role in many communities, especially rural ones. Most villages and all towns have a local post office. This is not only a centralized posting point for letters and parcels, but also a place for purchasing items such as postcards, birthday cards and stamps. It holds 
copies of most government and is often the only localized point for obtaining them. Such forms include passport applications, vehicle registration, and driving licence applications. Many post offices also offer banking facilities and are the collection point for most government benefits, such as state pensions and social security payments. There is also a continuing drive for post offices to offer new services to customers. This is especially true in rural areas where many banks and other local businesses are closing and their roles are being taken on by the post offices.

Consequently, the local post office typically offers a range of unique and essential services to the local community. It is important that no-one is excluded from access to these services. With a very large proportion of pensioners visiting post offices to collect their pensions, the age profile of post office customers is concentrated in the over-65's. Thus with the increased variability in capability associated with older customers, the potential for excluding large numbers of TPO customers from services that are not designed for maximum inclusivity is very great.

The development of product and service interfaces that offer universal access is clearly an essential component in facilitating a genuinely inclusive environment in all post offices and this paper highlights the many issues facing employers and service providers intent on creating such conditions.

To achieve this goal, though, it is necessary to focus first on the users and their needs and then reflect on the needs of the designer. This paper shall illustrate this by discussing the application of a five level design approach to the review of a joint development of an Information Point between TPO, a supplier and the authors.

\section{DESIGN STRATEGIES}

When designing any new product, it is necessary to adopt a three stage development strategy:

- $\quad$ Stage 1 - define the problem - gain an understanding of the system requirements;

- Stage 2 - develop a solution - develop a system that includes consideration of able-bodied and motionimpaired users;

- Stage 3 - evaluate the solution - make sure that the solution is effective.

There are two principal approaches to designing an interface for different user capabilities. The first is to design for a particular application and then tailor it retrospectively to different users. The second is to change the definition of the user at the very outset of the design process to include a wider range of capabilities. These approaches can be described as adaptive and proactive respectively [16]. The inclusion of a broader range of user capabilities affects all three of the above stages:

- Stage 1 - the problem definition should explicitly include reference to the intended target users;
- Stage 2 - an appropriate design approach for the target users should be adopted;

- Stage 3 - the target users should be included in the evaluation process.

\section{Design For All Approaches}

A number of design approaches exist for users with impairments, irrespective of whether caused by aging or congenital conditions. The approaches can broadly be defined by the target user groups and how they aim to enable usability. For instance, most inclusive design approaches define the target population as being older users, functionally impaired users or the whole population. One of the most common methods of dividing the approaches is the design method by which they aim to allow accessibility by those with limited capability. One common approach is to design specific solutions for specific needs.

For example, rehabilitation design [9] focuses on developing solutions for specific impairments. It has been claimed that the resultant products are not meeting the needs of elderly [8] or functionally impaired users [4] and this is reflected in poor sales figures [12].

Design by story-telling [9] is a similar approach, but this time with a four stage structure: (i) understand what it is like being old; (ii) observe what old people do and how they cope; (iii) visualize a different scenario without technical constraint; (iv) evaluate a product for the intended users. This approach puts the emphasis on age, as if that is the source of difficulties, instead of appreciating that the only difficulties truly presented by advancing years is the increasing prevalence of impairments. Also, it is difficult, maybe even presumptuous, to try to understand what it is like being old. Understanding the effects of being impaired, would be better.

Both of the above methods focus on either the elderly or the disabled as minority groups to be catered for by their own special needs products to specifically ameliorate their impairments. This leads to niche products that often suffer from branding and stigmatism problems. It is more acceptable to produce products that are inclusive without labeling the users.

Transgenerational design adopts the stance that products should be designed from the outset to avoid unnecessary accessibility problems on the grounds of age, by including users of all ages in the design process [15]. However, this still puts the emphasis on age rather than capability.

Universal design [9] attempts to maximize the number of people for whom a product is accessible and usable. It achieves this by trying to make the user base as broad as possible. This is a laudable approach that stops people being excluded by arbitrary and ill-informed design decisions simply because different user capabilities were not considered during the design process. However, a key question that needs to be addressed is how inclusive 'universal' really is. Owing to the huge diversity of people 
and their capabilities, it is unlikely that a single product solution will be accessible by everyone.

The user pyramid design approach reflects the wide range of user capabilities and their impact on the design process by categorizing the target user capabilities [3]. Here the user population is described as being in three broad bands. The able-bodied/fully capable make up the base of the pyramid, those with, say, reduced strength and mobility comprise the middle layer and the severely impaired occupy the peak. The approach claims that if products are designed to be accessible by a particular layer, then the resultant product will be accessible by those with less severe or no impairments. This is an important re-think of inclusive design approaches as it allows for both the possibility of different products for different people, and also the notion that products can reach across boundaries of capability. It is worth noting, though, that some of the filtered-down designs may be sub-optimal for more capable users.

\section{The Inclusive Design Cube}

Building on the concept of the user pyramid with its banding of users by impairment level, the authors have developed a model that relates capability level, population profile and suitable design approach in a simple graphical format. The resultant model, referred to as the inclusive design cube (IDC) [10], is shown in Figure 1. Each axis on the cube represents user capability and the enclosed volumes reflect population coverage

The user pyramid approach contends that there are three principal levels of capability: (i) severely impaired, (ii) moderately impaired and (iii) unimpaired. The user cube extends this concept more explicitly with three design approaches. It was recognized that the principles of universal design would generate products that would be widely accessible by large sections of the population and hence have good population coverage. Consequently this approach, denoted user-aware design, dominates the volume of the cube. However, it was also noted that the resultant products would be unlikely to be accessible to the more severely impaired/less capable.

For the most severely impaired users, it may be necessary to adopt rehabilitation design approaches of designing custom products for specific users. This is labeled special purpose design in Figure 1.

In between the two approaches is an intermediary design approach that has potentially flexible boundaries. Modular or rapid/mass customizable design takes a base unit designed using the user aware design principles, but with a changeable interface that is either adaptable or can be swapped for one of a series of modular designs.

The Inclusive Design Cube is a very potent visualization tool and communicates the needs of different sections of the population. However, for practical implementation of inclusive design practices, it is necessary to have a rigorous methodology.
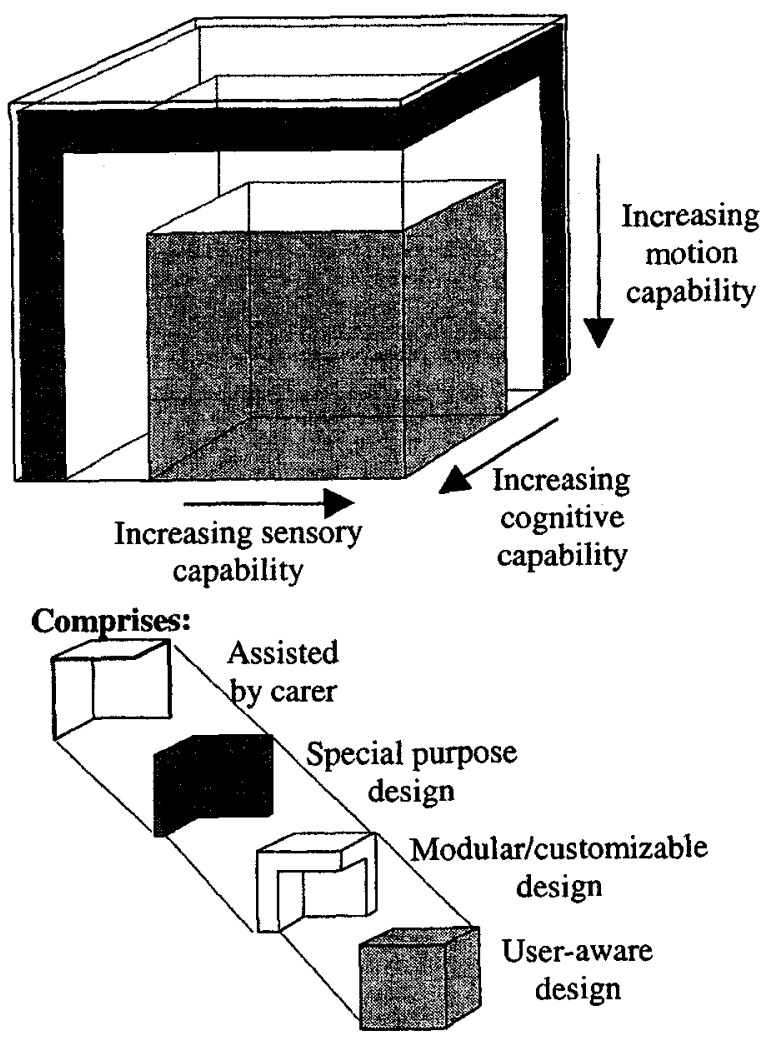

Figure 1. The Inclusive Design Cube (IDC).

\section{THE DESIGN METHODOLOGY}

In order to produce a usable and accessible product or service, it is necessary to adopt strongly user-centered design practices. It is important to be able to modify and refine the interface iteratively, combining both design steps and usability evaluations, which typically involve measurement against known performance criteria. Nielsen gives an account of the use of such criteria in a method known as heuristic evaluation [14].

Developing a usable product or service interface for a wider range of user capabilities involves understanding the fundamental nature of the interaction. Typical interaction with an interface consists of the user perceiving an output from the product, deciding a course of action and the implementing the response. These steps can be explicitly identified as perception, cognition and motor actions [5] and relate directly to the user's sensory, cognitive and motor capabilities respectively. Three of Nielsen's heuristics explicitly address these functions:

- Visibility of system status - the user must be given sufficient feedback to gain a clear understanding of the current state of the complete system;

- Match between system and real world - the system must accurately follow the user's intentions;

- User control and freedom - the user must be given suitably intuitive and versatile controls for clear and succinct communication of intent. 
Each of these heuristics effectively addresses the perceptual cognitive and motor functions of the user. Building on these heuristics, a design approach has been developed that expands the second stage of the design process, solution development, into three specified steps [11]. Each level of the resultant design approach, shown in Figure 2 , is accompanied by user trials throughout and a final evaluation period before progression to the next level, thus providing a framework with clearly defined goals for system usability. The 5 level approach has been successfully applied to the design of a software interface for the control of an assistive technology robot [6].

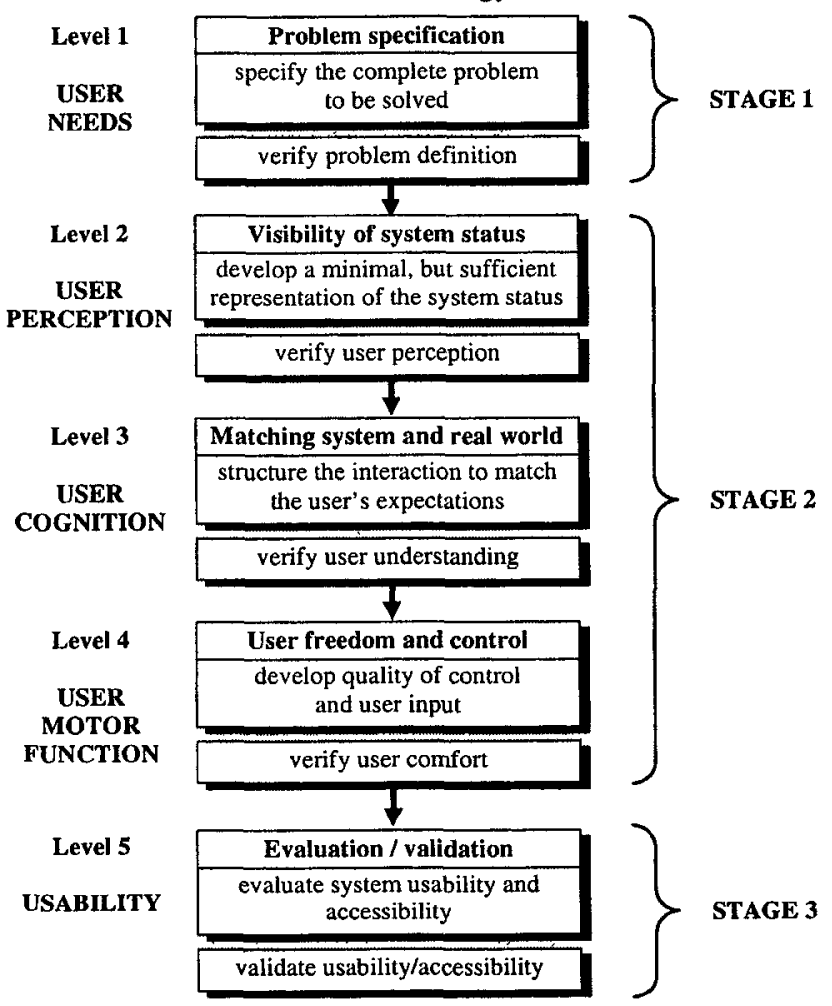

Figure 2. The 5 level design approach.

Both the 5 level approach and the Inclusive Design Cube share the same inherent emphasis on the interaction consisting of perceptual, cognitive and motor actions. It is therefore possible to combine the two during the design process.

Taking the 5 level approach as the framework for the development, the IDC can be adapted to monitor the progress of the design by indicating the population coverage achieved by different design choices. Effectively, the 5 level approach can be thought of as designing for each axis on the cube. The modification necessary to use the IDC for this is a straightforward re-labeling of the axes to reflect Levels 2 to 4 of the 5 level design approach. The resultant Inclusive Design Cube is shown in Figure 3.

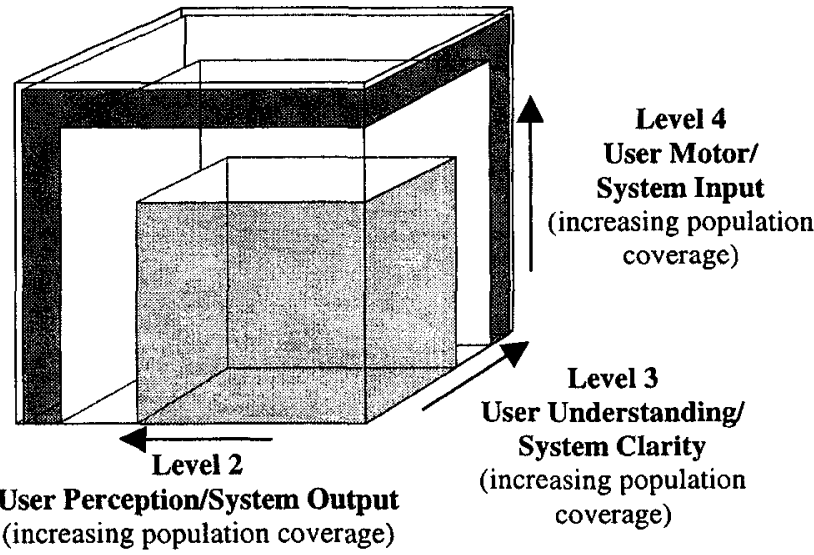

Figure 3. The IDC for use with the 5 level approach.

\section{CASE STUDY: DESIGNING AN INFORMATION POINT}

The Post Office (TPO) is very interested in introducing automated customer information points (IPs) into their post offices to augment their current range of services. TPO is also very keen to ensure that the product is as usable and accessible as possible, not simply for DDA compliance, but also to engender customer satisfaction. An initial concept system has been developed by a design company for review as a possible solution to TPO's requirements for an information point. The concept system is shown in Figure 4.

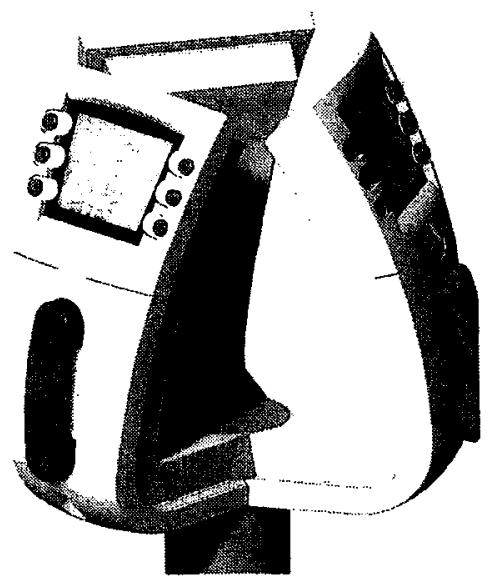

Figure 4. The concept Information Point.

The concept IP has been developed using traditional product design approaches that dominate industrial design and focus as much on style as function. It is principally a technological solution based on examining the required technological components for possible customer transactions using the IP. The purpose of the case study here is to examine the effectiveness and usability of the prototype and demonstrate how application of the 5 level model would have affected its overall usability. The aim is to provide guidelines for re-designing the concept IP using user-aware design principles to maximize the number of potential users.

The prototype IP consists of three display heads mounted on a single pedestal of fixed height. The unit is designed to 
be free-standing and possess a small footprint. Each unit head has a small LCD panel mounted at a fixed angle, with two columns of three buttons on either side of the screen and a telephone handset for audio output. The output is primarily recorded video footage with accompanying soundtrack available via the handset. Input is achieved through the six buttons.

As a comparison, the functionality and usability of the prototype IP shall be compared to a low-end, internet café style PC. That is not to suggest that a PC is the most viable alternative, simply that it gives a good, and widely understood, comparison of the capabilities of other technologies available for a similar purchasing price.

\section{The design approach for the IP}

Adapting the five level model for the information point, the design process can be sub-divided into the following steps:

Level 1 What are the IP system requirements?

Level 2 How does the user receive information from it?

Level 3 Does the user understand what is happening?

Level 4 How does the user enter information into the IP and what are the physical demands on the user?

Level 5 Evaluation of the IP

\section{Level 1: System requirements}

The first step in the design process is to identify the system requirements. This can be thought of as the following process:

$$
\begin{gathered}
\text { product } \\
\text { objectives }
\end{gathered}+\begin{gathered}
\text { potential } \\
\text { users }
\end{gathered} \Rightarrow \begin{gathered}
\text { specific } \\
\text { requirements }
\end{gathered}
$$

In this case this involves determining the IP objectives and establishing the target users.

\section{Level 1 evaluation}

For this case study, the IP objectives are to reduce queue length for the counter service; improve customer service; and enhance the environment and services in post offices. These objectives must be achieved in such a way that meets legislated requirements, such as the DDA and hopefully be a profitable venture.

Expanding the objectives highlights a range of potential activities and design requirements that need to be supported. For example, to meet the legislated requirements that all services are equally accessible to all customers, the needs of the functionally impaired need to be considered. A more inclusive set of design requirements needs to be identified.

Reduction in queue length can be obtained by offering typical counter services through the IP. However, identifying which services are to be supported will influence the overall IP design. The required baseline functionality includes supporting: web browsing of governmental information sites; simple transactions - e.g. purchasing stamps - requires the ability to accept money or credit cards; and providing access to TPO information - e.g. guides on which forms to collect for vehicle registration or buying a television licence. Other desirable actions include more complex transactions, such as banking, and on-line form completion and submission. Some of the interactions could be quite time-consuming and so thought needs to be given to the expected time duration of use.

Improvements in customer service can be achieved by the reduction in queue lengths. However, the IP can also improve service by offering existing services in a new format and also offering completely new services. This should also enhance the post office environment and services by offering streamlining of access to services.

The target user of the new Information Point is the typical post office customer. The immediate image of a typical customer, particularly one who is likely to use the IP, is usually a young and able-bodied man. However, owing to the age profile of typical post office customers, the real target person is likely to be older and have at least one major impairment, most likely visual, auditory or motor. The typical customer is also likely to exhibit behavior somewhere between two user-type extremes that can be thought of as techno-phobes and techno-philes.

Techno-phobes require that the system be as nonthreatening and inviting as possible. The on-screen instructions would have to be helpful and not use intimidating language. A powerful incentive to use the new device, instead of queuing for the counter service, would be needed to encourage this user to use the IP. Such users could also be discouraged from future ventures into new technology if the experience proves to be unrewarding. Techno-philes, however, usually require little encouragement to play with a new toy. In terms of reducing queue length, the IP could work simply by enticing the techno-philes out of the queue.

For the typical customer a careful balance that attracts both the techno-phobes and the techno-philes would be required.

\section{Level 1 summary}

All of the above discussion, both regarding objectives and users, should be independent of the nature of the system to be implemented. For maximum accessibility and legislative compliance, this process should be entirely driven by user needs and product objectives. However, the concept IP was developed with technological considerations as the principal focus. Therefore, the results of this level imply that the concept IP may not offer the necessary functionality or usability because these issues have not been explicitly defined by TPO at this stage. This will be examined further in the following levels of the methodology.

\section{Level 2 - User output}

Output to the user typically takes one of three forms for a computer type system:

1. Visual symbolic feedback - typically shapes, colours and icons, but could also include sign language; 
2. Visual textual feedback - written text;

3. Audio feedback - spoken text.

Visual symbolic feedback is often very immediate, but is difficult to describe to those with impaired vision and can be very misleading if implemented badly. Users can be quickly deterred by being required to recognize meaningless icons. If this method is to be implemented, then careful consideration needs to be given to the meanings of the symbols or icons used.

Visual textual feedback is not as immediate as symbolic, a picture sometimes really is worth a thousand words, but is relatively unambiguous. Such feedback requires the cognitive and physical ability to read, however it does lend itself to being complemented by audio or symbolic output. Relying on visual output alone risks excluding the 1.7 million adults in the UK with visual impairments [13].

Volume adjustable audio output avoids excluding those with visual impairments. Audio output is naturally sequential, though, and potentially less efficient than visual output because multiple options cannot be presented simultaneously. There are also 3 million hearing impaired people who could potentially be excluded if audio output was the only channel [13]. This is a conservative estimate that does not include those whose hearing would be environmentally impaired if the post office is noisy.

Used individually, in a suitable environment, each of the output methods described should provide enough feedback for a user with unimpaired hearing or sight. However, a post office is not an ideal environment for computer usage, and the users cannot be assumed to have ideal hearing or sight. Consequently, using the principle of user-aware design from the IDC, i.e. to push the boundaries of the basic design to include as many people as possible, a mix of output measures would provide the best population coverage. For instance, the inclusion of text subtitles to supplement audio output would reduce the number of potential users being excluded.

\section{Level 2 evaluation}

The other major concern when addressing user output is that the output solution chosen is positioned in an accessible manner. The number of people excluded by the fixed height and angle of the concept IP display is a good example of this. The display viewing height is set rather high at $1610 \mathrm{~mm}$, with a lower bound on the viewing height of $1451 \mathrm{~mm}$. As shown in Figure 5, this excludes half of women of 65 and approximately a quarter of all adult women, rendering 6 million women unable to view the display [1].

Both the PC option and the IP prototype are capable of providing support for all three types of output, although the prototype IP currently only offers visual symbolic and audio output. The PC model offers the additional benefits of a larger and movable screen, whereas that of the IP is smaller and immovable.
Level 2 summary

Relying solely on audio output would exclude 1.7 million people and using visual output only would exclude 3 million. The concept IP currently excludes 6 million people by being positioned too high and not being adjustable.

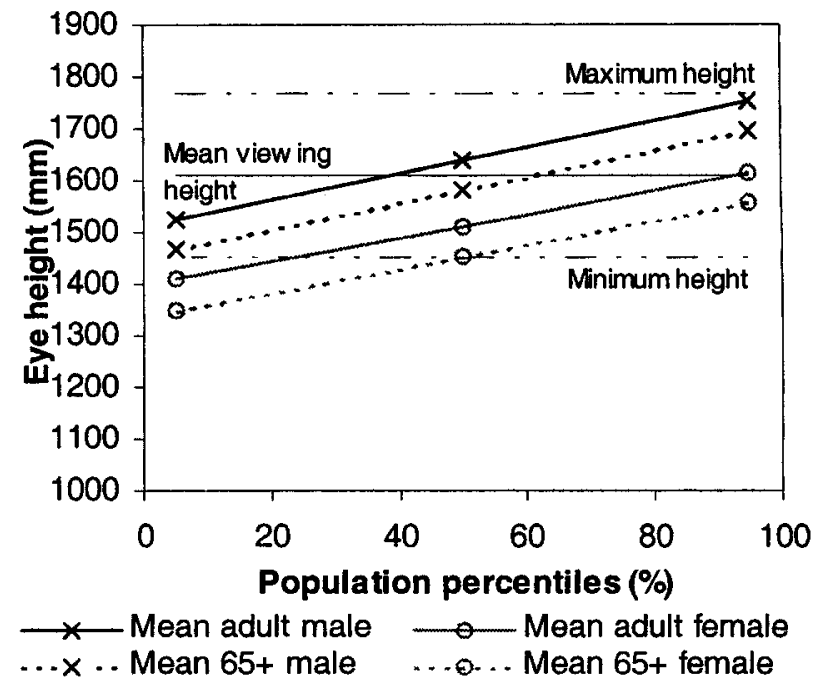

Figure 5. Adult eye height distributions.

Level 3 - User understanding/cognition

With this process being an evaluation of the physical accessibility of the concept IP it is impossible to examine the usability of the software interface, and hence user understanding, at this stage. A discussion of the required development process is presented instead.

The design of the interface for maximum user understanding and minimum cognitive load is an essential component of the successful development of a truly usable and accessible product. Matching the behavior of the IP to the user's expectation of what the system should be doing relies on appropriate output methods having been identified and implemented from Level 2. With an appropriate feedback system in place, it is possible to begin structuring the interaction to maximize the user's understanding of the processes being performed and any input required.

To achieve this, it is necessary to implement usability engineering techniques such as cognitive walkthrough[5] where identified tasks are sub-divided into their simplest component sub-tasks that can be quantified and measured, or heuristic evaluation [14].

\section{Level 3 evaluation}

Looking only at the output capabilities, both the IP and the PC options could have broadly similar interface structures for those tasks that both options support, although the increased display area of the PC monitor offers the potential for richer output.

Level 3 summary

The structure of the interaction, and hence of the interface, is partly dependent upon input and output modes chosen. 
Under the 5 level approach, only the output modes will have been specified by this stage. However, with the focus on the technology in the design of the concept IP and the decision to include only six buttons for input, the functionality of the software interface is arbitrarily limited to selection from a list. However, if the list is well designed, then the system has the potential to at least be easy to learn.

\section{Level 4 - User input/comfort}

Having established the required information flow from the user to the processor, it is now possible to examine the options for facilitating that. Typically available input options include: voice recognition; touch-screens; buttons or switches; analogue pointing devices, e.g. mouse or trackball; keyboards; other technologies, e.g. smart cards.

Untrained voice recognition with such a potentially large and specialized vocabulary, such as names and addresses, is still technologically suspect. This problem could be exacerbated by a noisy environment of use. However, if this limitation could be overcome then voice recognition offers a powerful input method.

Touch-screens are easy to learn, but unless placed at a comfortable height and orientation may prove tiring to use over a period of time, especially for older users. They are also difficult to use by visually impaired users, so cannot form the only input method, but could potentially be used in conjunction with other input modes.

If the software interface is well designed, the use of buttons and switches is also easy to learn. However, the functionality offered is typically limited to selection from a list of options. This may prove to be too restrictive for activities such as web-surfing or text entry.

Analogue pointing devices offer a robust input method, but require the ability to use a cursor. Consequently, those unfamiliar with such devices, those with limited motor capability or those with impaired vision may struggle to use them.

Keyboards are probably the most versatile and powerful entry tool, although they do present difficulties to those with impaired motor or visual function and can be intimidating to those not familiar with them.

\section{Level 4 evaluation}

The prototype IP only offers button input through a limited number of buttons. Text entry is impossible without the use of voice recognition technology or scanning input to a keyboard emulator. Some text entry requirements could be circumvented, though, by the use of smart cards that contained information about the user and could be inserted into the IP.

In comparison, the PC option offers much more freedom for users. The keyboard and mouse arrangement certainly has its usability and accessibility problems, but does offer a rich medium for data entry. The ability to substitute different input channels, such as keyboard emulators if the keyboard presents difficulties, offers a significant degree of flexibility. Substitution of input devices is more in line with modular design rather than user aware design, but offering redundancy in the choice of inputs is a valid user-aware design strategy.

The other issue of major concern is the physical positioning of the input for user comfort. The free-standing design of the prototype is likely to affect anyone who has difficulty standing for extended periods of time. The requirement that both hands be used eliminates anyone who needs to use a walking stick for support, some $57 \%$ of the over 60 's who make up the majority to TPO's customers. Across the whole UK population, 10.7 million people use walking sticks and would be unable to use the prototype for any extended period of time. The associated reaching and dexterity requirements for operating the buttons and the handset also excludes a further 3 million people [13].

\section{Level 4 summary}

The concept IP will exclude 10.7 million people who have difficulty standing for extended periods of time and 3 million people who cannot operate the buttons and handset.

\section{Level 5 - Evaluation}

This review process represents the first stage of the evaluation of the concept IP, which is now entering a phase of re-design. Once this process is complete, units shall be deployed in a number of post offices and their use observed. The evaluation of the IP shall involve the identification of appropriate measures of success. These will reflect the initial objectives of the IP design.

\section{DISCUSSION}

Having applied a first pass of the 5-level approach as a review, a number of pointers for the re-design of the prototype have been identified. Starting with Level 1 , it is clear that the existing design does not offer sufficient functionality to meet TPOs requirements.

Levels 2 to 4 illustrate the importance of being aware of the user needs throughout the design process. Assuming that impairments are independently distributed across the population and that the presence of one impairment does not alter the probability of another impairment being present, approximately 21 million potential users could have been excluded from using the IP because of the failure to consider different user physical capabilities at the outset. That is $45 \%$ of the total UK adult population and does not include those who may be environmentally impaired, for example through background noise or glare from the display, or temporarily impaired through injury. To make matters worse, this number is likely to increase as the years go by, because the proportion of the population who are over 65 , and hence statistically more likely to exhibit the impairments identified as causing difficulty in using the unit, is increasing.

The case study, in identifying the scale of exclusion, also shows how important it is to implement a structured 
approach to the user-aware design process. By identifying the interaction along the lines of perception, cognition and motor functions, the required user sensory cognitive and motor capabilities can be identified easily. It also drives home how arbitrary design choices, such as requiring the user to stand, can exclude vast swathes of the population. The Inclusive Design Cube in Figure 6 shows the differences in the number of people included by the current design and be a re-design using user-aware design methods.
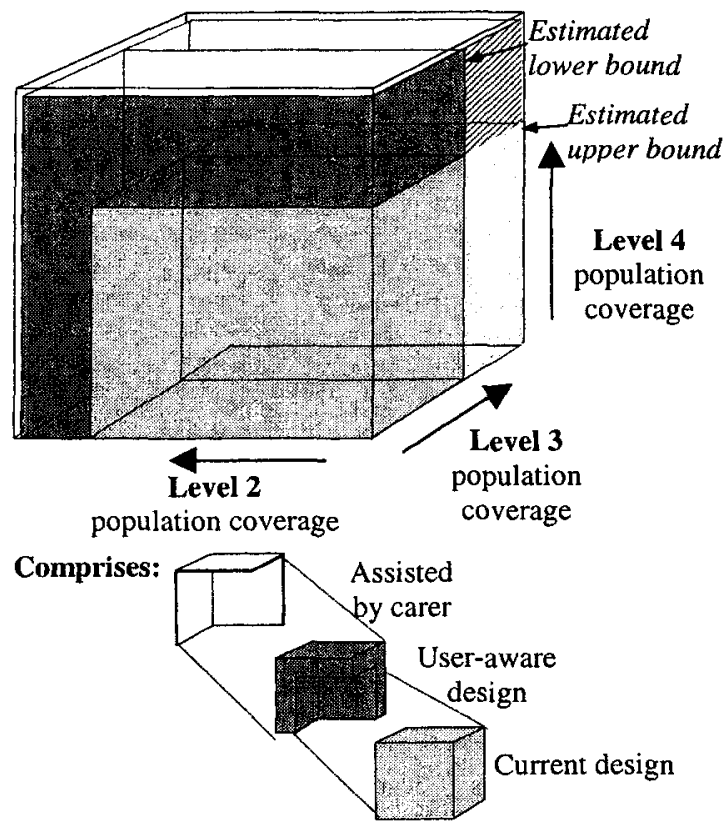

Figure 6. The current design compared to user-aware design.

Fortunately, potential, and often simple, remedies can also be identified. For instance placing the unit at desk height and having chairs nearby would remove almost all of the difficulties for the 17.8 million people with different motor capabilities and physical properties from the designer's target user.

As a final note, this paper has shown the level of exclusion that can be avoided through the application of a methodical user centered design approach such as the five level model and highlights areas where the greatest benefit can be achieved. Therefore, the real challenge that this research identifies is how to make industrial designers more aware of these potential benefits.

\section{FURTHER WORK}

We are currently assisting TPO and the supplier in the design of a new IP prototype based on the results of the user-aware design approach. The revised prototype should be completed before the end of the year.

\section{ACKNOWLEDGMENTS}

We would like to thank Dr Joanne Coy of The Post Office Research Group for her assistance with this paper and TPO and the EPSRC for funding this research.

\section{REFERENCES}

1. ADULTDATA - The handbook of adult anthropometric and strength measurements, Department of Trade and Industry, 1998.

2. Americans with Disabilities Act of 1990, US Public Law 101-336, USA, 1990.

3. Benktzon, M. Designing for our future selves: the Swedish experience. Applied Ergonomics 24, 1, (1993), 19-27.

4. Buhler, C. Robotics for rehabilitation - a European(?) perspective. Robotica 16, 5, (1998), 487-490.

5. Card, S.K., Moran, T.P., and Newell, A. The Psychology of Human-Computer Interaction. Lawrence Erlbaum Associates, Inc., 1983.

6. Clarkson, P.J., Keates, S., Dowland, R. The design and control of assistive devices, in Proceedings of the International Conference on Engineering Design (ICED) '99, (Munich, Germany, August 1999), Vol. 1, 425-428.

7. The Disability Discrimination Act, Department for Education and Employment, UK, 1996.

8. Gardner, L., Powell, L., and Page, M. An appraisal of a selection of products currently available to older consumers. Applied Ergonomics 24, 1, (1993), 35-39.

9. Hewer, S., et al. The DAN teaching pack: Incorporating age-related issues into design courses. RSA, London., 1995.

10. Keates, S., and Clarkson, P.J. Towards a generic approach for designing for all users, in Proceedings of RESNA '99, (Long Beach, CA, June 1999), pp97-99.

11. Keates, S., Clarkson, P.J., and Robinson, P. A design approach for accessibility. Human-Computer Interaction. Lawrence Erlbaum Associates, Vol. 2 (Bullinger and Ziegler eds 1999), 878-882.

12. Mahoney, R. Robotic products for rehabilitation: Status and strategy, in Proceedings of ICORR '97, (Bath, UK, April 1997), 12-22.

13. Martin, J., Meltzer, H., and Elliot, D. OPCS surveys of disability in Great Britain. Report 1: The prevalence of disability among adults, HMSO, 1988.

14. Nielsen, J., and Mack, R.L. Usability Inspection Methods. Wiley \& Sons, 1993.

15. Pirkl, J. Transgenerational Design: Products for an aging population, Van Nostrand Reinhold, NY, 1993.

16. Stary, $C$. The role of design and evaluation principles for user interfaces for all, in Proceedings of $\mathrm{HCI}$ International '97, (San Francisco, CA, August, 1997), 477-480. 